ACCEPTED MANUSCRIPT

\title{
Scaling dynamics of globule-to-coil phase transition in double-network hydrogel with ultra-high stretchable strength
}

To cite this article before publication: Haibao Lu et al 2020 Smart Mater. Struct. in press https://doi.org/10.1088/1361-665X/ab9e0c

\section{Manuscript version: Accepted Manuscript}

Accepted Manuscript is "the version of the article accepted for publication including all changes made as a result of the peer review process, and which may also include the addition to the article by IOP Publishing of a header, an article ID, a cover sheet and/or an 'Accepted Manuscript' watermark, but excluding any other editing, typesetting or other changes made by IOP Publishing and/or its licensors"

This Accepted Manuscript is @ 2020 IOP Publishing Ltd.

During the embargo period (the 12 month period from the publication of the Version of Record of this article), the Accepted Manuscript is fully protected by copyright and cannot be reused or reposted elsewhere.

As the Version of Record of this article is going to be / has been published on a subscription basis, this Accepted Manuscript is available for reuse under a CC BY-NC-ND 3.0 licence after the 12 month embargo period.

After the embargo period, everyone is permitted to use copy and redistribute this article for non-commercial purposes only, provided that they adhere to all the terms of the licence https://creativecommons.org/licences/by-nc-nd/3.0

Although reasonable endeavours have been taken to obtain all necessary permissions from third parties to include their copyrighted content within this article, their full citation and copyright line may not be present in this Accepted Manuscript version. Before using any content from this article, please refer to the Version of Record on IOPscience once published for full citation and copyright details, as permissions will likely be required. All third party content is fully copyright protected, unless specifically stated otherwise in the figure caption in the Version of Record.

View the article online for updates and enhancements. 


\section{Scaling dynamics of globule-to-coil phase transition in double-network hydrogel with ultra-high stretchable strength}

Haibao Lu ${ }^{1,3}$, Ziyu Xing ${ }^{1}$, Mokarram Hossain ${ }^{2}$ and Jinsong Leng ${ }^{1}$

${ }^{1}$ Science and Technology on Advanced Composites in Special Environments Laboratory, Harbin Institute of Technology, Harbin 150080, China

${ }^{2}$ Zienkiewicz Centre for Computational Engineering, College of Engineering, Swansea University, Swansea, UK

${ }^{3}$ Corresponding author, E-mail: $\underline{\text { uhb@ } @ \text { hit.edu.cn }}$

Abstract: Temperature-induced globule-to-coil transition in polymers has well been studied, and it is dependent strongly on temperature in their solutions due to significantly conformational changes of polymer molecules. In this study, a scaling framework is firstly developed to investigate stress-induced globule-to-coil transition in double-network (DN) hydrogels with an ultra-high stretchable strength. Free energy and globule radius functions are introduced to formulate the constitutive relationship of the DN hydrogels, in which stress-induced swelling and globule-to-coil transition are described for the hydrotropic and relatively hydrophobic networks, respectively. A cooperative free energy model based on the Flory-Huggins solution theory is then proposed for the DN hydrogels. The effectiveness of model is demonstrated by applying it to predict stress-induced globule-to-coil transitions in DN hydrogels and elastomers, where the theoretical results show good agreements with the experimental ones. We expect this study explores the scaling dynamics and provides an effective guidance on designing advanced ultra-high mechanical performance in DN hydrogel and elastomer. 
Keywords: double-network hydrogel, globule-coil transition, cooperative dynamics

\section{Introduction}

Hydrogel is a network of polymer chains, which are connected by crosslinks and swollen by absorbing solvent when placed in an aqueous solution (solvation) $[1,2]$. The crosslinks may be neutral and ionic interactions between polyelectrolyte chains, therefore, the hydrogels are generally classified into two categories, i.e. neutral hydrogels and ionic hydrogels $[3,4]$. Hydrogel is one type of stimulus-responsive materials, which are featured by their highly stretchable behaviours through their absorbent polymeric networks, which contain over $90 \%$, water [5-8]. In terms of thermodynamics of polymer solution, hydrogels are normally rubbery-like soft matters, and thus respond to the external stress in an elastic manner [9-12]. Therefore, mechanical and thermomechanical behaviours of the hydrogels have been generally studied using the rubber elasticity theory and free energy equation [13-19]. A significant development has been achieved in this field to reveal the feasibility to reproduce the constitutive stress-strain relationships of the hydrogels using elastic and mixing free energy functions [20-24]. Good agreements between the theoretical prediction and experimental results for the hydrogels have been reported [25-31].

Recently, double-network (DN) hydrogels have been proposed and received great attention due to their ultra-high mechanical strength and toughness [16-19]. The conventional hydrogels are easily broken even at a low stress, and thus present soft and weak mechanical properties [26-31]. DN hydrogels are combinations of two types of 
polymeric networks, in which one relatively hydrophobic network is able to resist to mechanical loading through breaking-up of network bonds, while the other hydrophilic network is highly stretchable [32-34]. Due to the opposite physical properties of these two types of networks, the DN hydrogels often undergo cooperative and complex conformational changes when they are under mechanical/mechanochemical loading [35-37]. However, the working mechanisms of mechanical deformations of the DN hydrogels have not been fully understood, and these are distinctly different from those of the conventional single-network ones.

Due to the complexity of their thermodynamics, in this study, a cooperative and theoretical model for the DN hydrogels are proposed, in which the stress-induced globule-to-coil transition of the relatively hydrophobic network has been identified as the driving force for the mechanical deformation of the DN hydrogels. Free energy and globule radius functions are introduced to formulate the constitutive relationships of the DN hydrogels, which are incorporated from a hydrophobic network and a hydrophilic network or two hydrophobic networks. The constitutive stress-strain relationship and thermomechanical behaviour are both studied and discussed using the Flory-Huggins solution theory. Finally, the simulation results of the proposed models are compared and verified using the experimental data reported in literature.

\section{Stress-induced globule-to-coil phase transition in DN hydrogel}

\subsection{Scaling dynamics and theoretical framework}

For DN hydrogels, the relatively hydrophobic and hydrophilic networks undergo distinct swelling behaviours, simultaneously. Therefore, it is necessary to investigate 
the swelling effects of water on DN hydrogels. According to the Flory-Huggins theory [38], the free energy equation per unit volume of DN hydrogel can be written as,

$$
\Delta F_{M}=R T\left(n_{1} \ln \phi_{1}+n_{2} \ln \phi_{2}+\chi n_{1} \phi_{2}\right)
$$

where $\Delta F_{M}$ is the mixing free energy per unit volume, $\chi$ is the Flory-Huggins interaction parameter, $n_{1}$ and $\phi_{1}$ represent the molar and volume fraction of water, respectively, $n_{2}$ and $\phi_{2}$ represent the molar and volume fraction of gel, respectively, $T$ is the temperature.

Meanwhile, the elastic free energy $\left(\Delta F_{e l}\right)$ per unit volume of DN hydrogels can be expressed as [38],

$$
\Delta F_{e l}=\frac{3 \xi k_{B} T}{2}\left[\left(\frac{1}{\phi_{2}}\right)^{2 / 3}-1\right]
$$

where $k_{B}$ is Boltzmann constant and $\xi$ refers to the number of chains.

The free energy function of DN hydrogels should be the sum of these two terms, e.g., $\Delta F=\Delta F_{M}+\Delta F_{e l}$, while $\phi_{1}=1-\phi_{2}=\frac{V}{V+V_{0}}, V+V_{0}=\lambda^{3}$ and $V_{0}=\lambda_{0}^{3}$. Finally, the energy function can therefore be obtained,

$$
\Delta F=-R \operatorname{Tn}_{1}\left[\ln \frac{\lambda^{3}-\lambda_{0}^{3}}{\lambda^{3}}+v \frac{\lambda^{3}-\lambda_{0}^{3}}{\lambda_{0}^{3}} \ln \frac{\lambda_{0}^{3}}{\lambda^{3}}+\left(B \frac{\lambda_{0}^{3}}{\lambda^{3}}+C\right) \frac{\lambda_{0}^{3}}{\lambda^{3}}\right]+\frac{3 \xi k T}{2}\left[\frac{\lambda^{2}}{\lambda_{0}^{2}}-1\right]
$$

where $\lambda_{0}$ is the initial elongation ratio, $\lambda$ is the final elongation ratio, $v$ is molar volume ratio of polymer to water and $R$ is the gas constant.

In DN hydrogels, the relatively hydrophobic network is assumed to undergo a globule-to-coil transition, while the hydrophilic network undergoes a swelling effect in response to the water, as shown in Figure 1. And the working mechanics of mechanical yielding is resulted from the stress-induced globule-to-coil transition of the relatively 
hydrophobic network in DN hydrogels. Initially, the globule radius of relatively hydrophobic network is decreased due to the osmotic pressure of the hydrophilic network, which is more able to mix with the water molecules. The mixing equilibrium equation of DN gels and inner water is incorporated from swelling of the hydrophilic network and a shrinking of the relatively hydrophobic network. Consequently, the osmotic pressure applied on the relatively hydrophobic network is gradually decreased due to the externally mechanical loading. Finally, the globule-to-coil transition of the relatively hydrophobic network is then induced by the mechanical loading, resulting from the globule radius gradually increased. Here the working mechanics of yielding is originated from the stress-induced globule-to-coil transition of the relatively hydrophobic network in DN hydrogels.

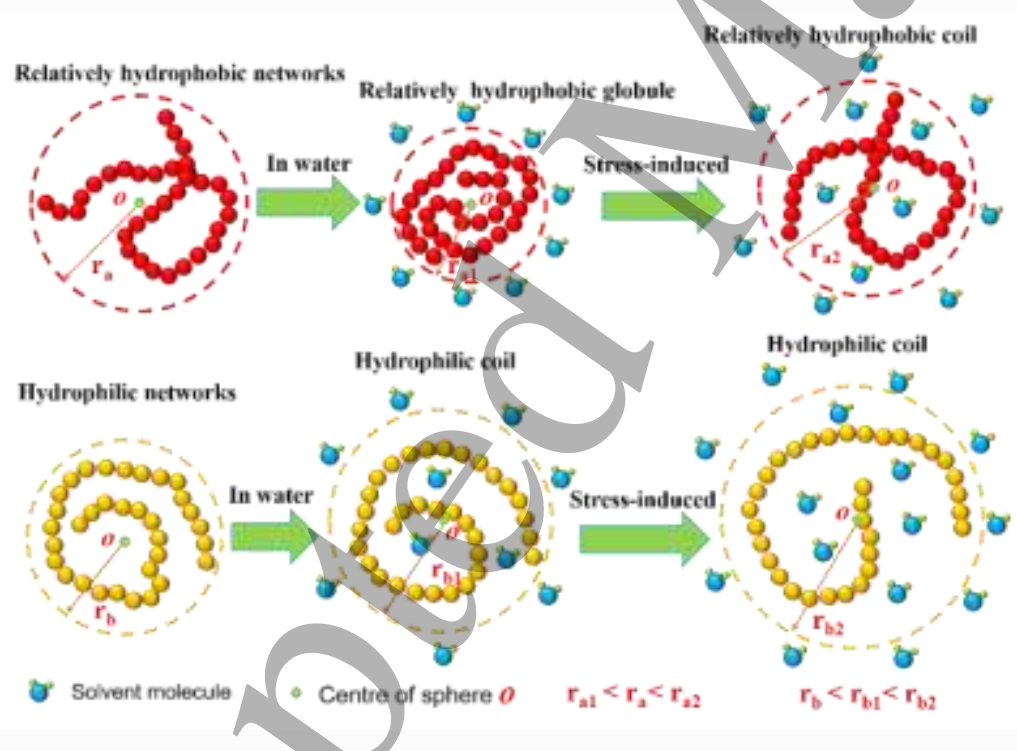

Figure 1. Schematic diagram of the globule-to-coil transition behaviours of the two networks in DN hydrogel.

Consequently, it is necessary to investigate the globule-to-coil transition in DN hydrogels. According to the previous study [38], the function of globule radius $\left(r_{b}\right)$ for the relatively hydrophobic network is expressed as, 


$$
r_{b} \propto b N_{B}^{\frac{2}{3}}\left(\gamma_{A B} b^{2} / T\right)
$$

where $b$ is the segment length, $N_{B}$ is the monomer number, $\gamma_{A B}$ is the interface energy between two networks and $T$ is temperature. On the other hand, we can use a thermodynamic parameter $\chi$ to describe the hydrogel swollen due to the water, and it is determined by the volume fraction of water $\left(\phi_{2}\right)$ as follows [38],

$$
\chi=B \phi_{2}+C \quad\left(\chi N_{B}=10.5\right)
$$

where $B$ and $C$ are given material constants. Substituting equation (5) into (4), the function of the outer radius of the globule $\left(r_{b}\right)$ is then obtained as,

$$
r_{b}=\frac{k_{r}}{\chi^{2 / 3}}=\frac{k_{r}}{\left(B \phi_{2}+C\right)^{2 / 3}}=\frac{k_{r}}{\left(B \frac{\lambda_{0}^{3}}{\lambda^{3}}+C\right)^{2 / 3}}
$$

where $k_{r}$ is a scaling constant for the globule of relatively hydrophobic network [39].

If a uniform stress is applied on the globule and DN hydrogels. The change in globule radius $\left(\Delta r_{b}\right)$ can be obtained by equation (6) [40],

$$
\Delta r_{b}=\frac{(1+\mu) r_{b}}{E}\left(\frac{\frac{1}{2}+\frac{1-2 \mu}{1+\mu} \frac{r_{b}^{3}}{r_{a}^{3}}}{\frac{r_{b}^{3}}{r_{a}^{3}}-1} q_{b}-\frac{\frac{1}{2}+\frac{1-2 \mu}{1+\mu}}{\frac{r_{b}^{3}}{r_{a}^{3}}-1} q_{a}\right)
$$

where $\mu$ is the Poísson's ratio which is taken as $\mu=0.49$ [40], $E$ is the elastic modulus, $r_{b}$ is the outer radius of the DN hydrogel, $r_{a}$ is the inner globule radius of the relatively hydrophobic network, $q_{a}$ and $q_{b}$ are the stresses applied on the globule and DN hydrogel, respectively. The swollen volume ratio $\left(\Delta V_{\text {mic }}\right)$ of globule is obtained as: 


$$
\Delta V_{\text {mic }}=\frac{\frac{4 \pi}{3}\left(r_{b}+\Delta r_{b}\right)^{3}-\frac{4 \pi}{3} r_{b}^{3}}{\frac{4 \pi}{3} r_{b}^{3}}=3 \frac{\Delta r_{b}}{r_{b}}+3\left(\frac{\Delta r_{b}}{r_{b}}\right)^{2}+\left(\frac{\Delta r_{b}}{r_{b}}\right)^{3}
$$

The elastic free energy of globule is therefore expressed as [38],

$$
\Delta F_{e l}^{m i c}=\frac{3 \xi k_{B} T}{2}\left[\frac{1}{\phi_{2}^{2 / 3}}\left(1+\Delta V_{m i c}\right)^{2 / 3}-1\right]
$$

In combination of equations (1) and (9), the free energy function of the DN hydrogel is finally obtained:

$$
\begin{aligned}
& \Delta F=\Delta F_{M}+\Delta F_{e l}^{m i c} \\
& =-R \operatorname{Tn}_{1}\left[\ln \frac{\lambda^{3}-\lambda_{0}^{3}}{\lambda^{3}}+v \frac{\lambda^{3}-\lambda_{0}^{3}}{\lambda_{0}^{3}} \ln \frac{\lambda_{0}^{3}}{\lambda^{3}}+\left(B \frac{\lambda_{0}^{3}}{\lambda^{3}}+C\right) \frac{\lambda_{0}^{3}}{\lambda^{3}}\right]+\frac{3 \xi k T}{2}\left[\frac{\lambda^{2}}{\lambda_{0}^{2}}\left(1+\Delta V_{m i c}\right)^{2 / 3}-1\right]
\end{aligned}
$$

According to the equation (10), the constitutive stress-strain/relationship of uniaxial tension can therefore be expressed:

$$
\begin{aligned}
& \sigma=\frac{\partial \Delta F}{\partial \lambda}=-R \operatorname{Tn}_{1}\left[\frac{3(1-v) \lambda_{0}^{3}}{\lambda\left(\lambda^{3}-\lambda_{0}^{3}\right)}+3 v \frac{3 \lambda^{2} \lambda_{0}^{3}}{\left(\lambda^{3}-\lambda_{0}^{3}\right)^{2}} \ln \frac{\lambda}{\lambda_{0}}-\left(6 B \frac{\lambda_{0}^{3}}{\lambda^{3}}+3 C\right) \frac{\lambda_{0}^{3}}{\lambda^{4}}\right] \\
& +R \operatorname{Tn}_{1} \frac{3 \xi}{N_{A} n_{1}} \frac{\lambda}{\lambda_{0}^{2}}\left(1+\Delta V_{\text {mic }}\right)^{2 / 3}+L
\end{aligned}
$$

where $L=R \operatorname{Tn}_{1} \frac{3 \xi}{2 N_{A} n_{1}} \frac{\lambda^{2}}{\lambda_{0}^{2}}\left[\left(1+\Delta V_{m i c}\right)^{2 / 3}\right]^{\prime}$.

Based on the proposed model, the analytical results are obtained and have been plotted in Figure 2. All the parameters used in equation (11) are listed in Table 1. These analytical results reveal that the stress is dramatically increased from $0 \mathrm{MPa}$ to 0.455 $\mathrm{MPa}$ as a function of dimensionless elongation ratio, which is increased from 1 to 1.25 , at a given constant of $r_{b} / r_{a}=5$. Meanwhile, the yielding strength is increased from $0.455 \mathrm{MPa}$ to $0.655 \mathrm{MPa}$ by increasing $r_{b} / r_{a}$ from 5 to 9 , at the same elongation ratio of $\lambda=1.25$. With an increase of the ratio of $r_{b} / r_{a}$, a higher free energy is necessary to enable the DN hydrogel to swell, while the free energy of globule is kept a constant. 
Here, a higher stress is therefore needed for the hydrogel to be maintained at the same elongation ratio, according to equations (10) and (11). These analytical results present a cooperative dynamics of DN hydrogels, in which there is a distinct difference in swelling behaviours of relatively hydrophobic and hydrophilic networks. Furthermore, the cooperative dynamics results in a complex mechanical behaviour of DN hydrogels.

Table 1. Values of parameters used in equation (11) for Figure 2.

\begin{tabular}{|c|c|c|c|c|c|c|c|c|c|c|}
\hline$\lambda_{0}$ & $B$ & $C$ & $v$ & $R T n_{1}(\mathrm{MPa})$ & $3 \xi / N_{A} n_{1}$ & $q_{a}$ & $E(\mathrm{MPa})$ & $L(\mathrm{MPa})$ & $r_{a}$ & $q_{b}$ \\
\hline 1 & 2 & 0.5 & 0.5 & 0.44 & 0.5 & 80 & 1 & 0.3 & 2 & 10 \\
\hline
\end{tabular}

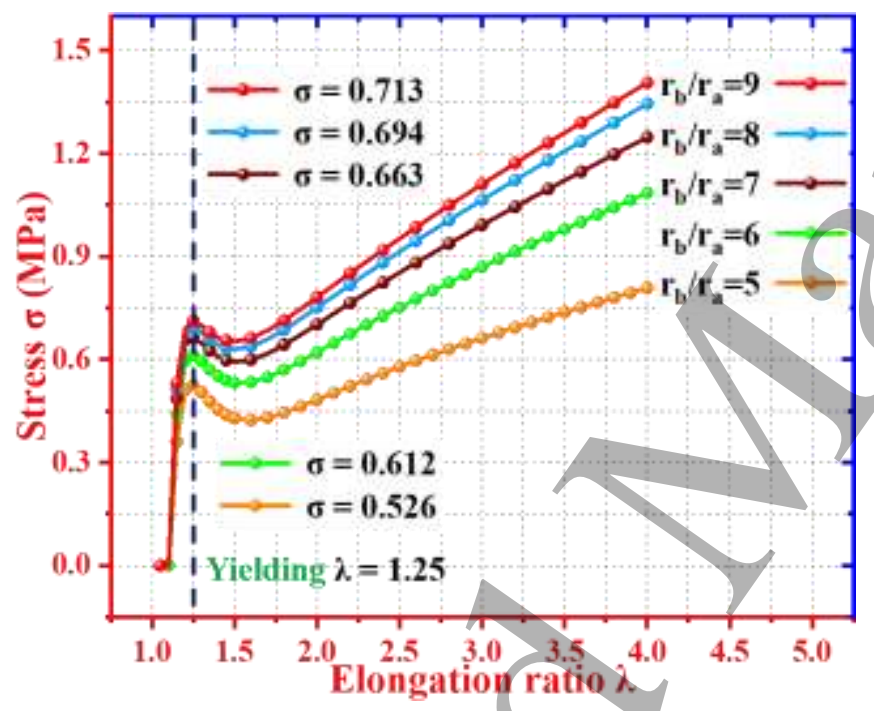

Figure 2. Simulation results of stress as a function of elongation ratio of DN hydrogel, at given $r_{b} / r_{a}$ ratios of $5,6,7,8$ and 9 .

On the other hand, the analytical results of the stress as a function of elongation ratio at a given molar fraction of water, i.e. $n_{1}=1 \times 10^{-4}, 1.5 \times 10^{-4}, 2 \times 10^{-4}, 2.5 \times 10^{-4}$ and $3 \times 10^{-}$ ${ }^{4}$ mol, have been obtained and plotted in Figure 3. The obtained fitting data are presented in Table 2. The simulation results show that the yielding stress of the hydrogel is gradually increased from $0.35 \mathrm{MPa}, 0.36 \mathrm{MPa}, 0.38 \mathrm{MPa}, 0.40 \mathrm{MPa}$ to $0.42 \mathrm{MPa}$ at a constant elongation ratio of $\lambda=1.24$, with the molar fraction increased from $1 \times 10^{-4}$ 
mol, $1.5 \times 10^{-4} \mathrm{~mol}, 2 \times 10^{-4} \mathrm{~mol}, 2.5 \times 10^{-4} \mathrm{~mol}$ to $3 \times 10^{-4} \mathrm{~mol}$. These simulation results reveal that the free energy of the gel is increased with the increase in molar fraction of solvent, thus resulting into an increase of the applied stress. Meanwhile, these analytical results also reveal that the minimum value of stress is decreased from $0.27 \mathrm{MPa}$ to 0.17 MPa with the molar fraction increased from $1 \times 10^{-4}$ mol to $3 \times 10^{-4} \mathrm{~mol}$. These results also reveal that the stress is indeed decreased with the elongation ratio increased from $\lambda=1.24$ to $\lambda=1.70$, which is mainly resulted from the globule-to-coil transition of the relatively hydrophobic network in DN hydrogels. A two-stage globule and coil transitions in DN gels have well characterized and predicted using our newly proposed model. In the process of globule transition, a certain amount of mechanical energy of the DN hydrogel is used to cause the relatively hydrophobic network compact, thus resulting in decrease of the free energy and stress. However, the free energy and stress are increased in the process of coil transition, because the relatively hydrophobic network absorbs the water to swell.

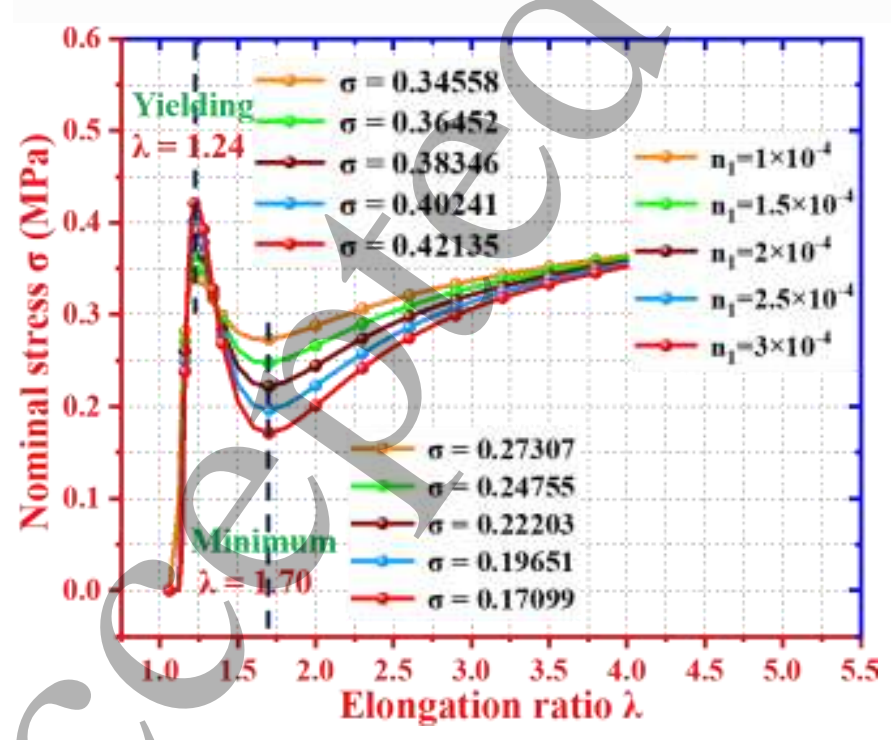

Figure 3. Analytical results of stress as a function of elongation ratio, at different molar fractions of water, i.e. $n_{1}=1 \times 10^{-4}, 1.5 \times 10^{-4}, 2 \times 10^{-4}, 2.5 \times 10^{-4}$ and $3 \times 10^{-4} \mathrm{~mol}$. 
Table 2. Values of parameters used in equation (11) for Figure 3.

\begin{tabular}{|c|c|c|c|c|c|c|c|c|c|c|c|}
\hline$\lambda_{0}$ & $k_{r}$ & $B$ & $C$ & $v$ & $R T(\mathrm{j} / \mathrm{mol})$ & $3 \xi / N_{A}(\mathrm{~mol})$ & $q_{a}$ & $E(\mathrm{MPa})$ & $L(\mathrm{MPa})$ & $r_{a}$ & $q_{b}$ \\
\hline 1 & 15 & 2 & 0.5 & 0.5 & $2.5 \times 10^{3}$ & $8 \times 10^{-6}$ & 80 & 1 & 0.3 & 2 & 10 \\
\hline
\end{tabular}

\subsection{Experimental verification of mechanical behaviours of DN hydrogels}

To verify the proposed model, four groups of experimental data [35] of the DN0.1, PVA-DN0.1, DN0.6 and PVA-DN0.6 have been employed to compare with the analytical results. For the PVA/PAMPS DN gels, the PAMPS (PAMPS: poly(2acrylamido-2-methylpropanesulfonic acid)) network is synthesized in the presence of poly(vinyl alcohol) (PVA) gels by an UV polymerization for eight hours [35]. Here, 0.1 and 0.6 are used to represent 0.1 and 0.6 in molar fractions of 2-oxoglutaric acid as the photoinitiator in the DN hydrogels. According to equation (11), the simulation results of stress as a function of elongation ratio were obtained and plotted in Figure 4. The stress of the DN hydrogel was gradually increased with the increase in tensile strain. All the parameters used in the equation (11) are listed in Table 3. After comparison, it was revealed that the simulation results were in well agreement with the experimental data of DN hydrogels. Furthermore, the yielding stresses were also predicted and characterized, where the stresses were $\sigma=0.79 \mathrm{MPa}, 0.74 \mathrm{MPa}, 0.58 \mathrm{MPa}$ and 0.47 MPa for the DN0.1, PVA-DN0.1, DN0.6 and PVA-DN0.6, respectively.

Table 3. Values of parameters used in equation (11) for DN0.1, PVA-DN0.1, DN0.6 and PVA-DN0.6, where $C=0.5, \quad r_{a}=2$ and $q_{b}=10$.

\begin{tabular}{|c|c|c|c|c|c|c|c|c|c|}
\hline & $\lambda_{0}$ & $k_{r}$ & $B$ & $v$ & $R T n_{1}(\mathrm{MPa})$ & $\frac{3 \xi}{N_{A} n_{1}}$ & $q_{a}$ & $E(\mathrm{MPa})$ & $L(\mathrm{MPa})$ \\
\hline DN0.1 & 10.5 & 14.9 & 1.97 & 0.47 & 0.94 & 0.28 & 110 & 0.74 & 0.5 \\
\hline PVA-DN0.1 & 10 & 15 & 2 & 0.46 & 0.8 & 0.23 & 109 & 0.75 & 0.41 \\
\hline
\end{tabular}




\begin{tabular}{|c|c|c|c|c|c|c|c|c|c|}
\hline DN0.6 & 9 & 14.8 & 2 & 0.75 & 0.6 & 0.28 & 102 & 0.69 & 0.53 \\
\hline PVA-DN0.6 & 9 & 14.8 & 1.92 & 0.55 & 0.44 & 0.21 & 96 & 0.57 & 0.41 \\
\hline
\end{tabular}

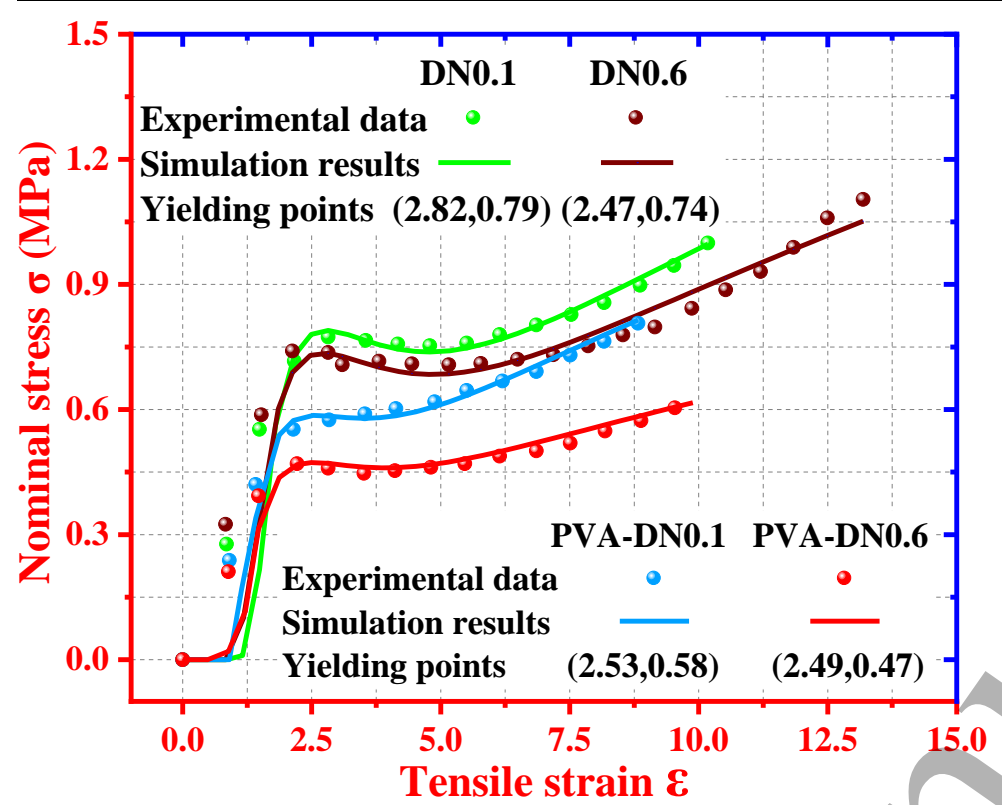

Figure 4. Comparisons between numerical results using equation (11) and experimental data [35] of the DN and PVA-DN hydrogels.

Furthermore, effect of water content $\left(C_{W}\right)$ on the mechanical behaviour of the DN hydrogel was also investigated, as it can be used to design and control of their mechanical behaviours by means of swelling effect. Figure 5 plots the constitutive stress-strain relationship of the hydrogel with various water contents. The obtained fitting data of equation (11) are presented in Table 4. Based on equation (11), it is found that the simulation results fit well with the experimental data of DN hydrogels with different water contents of $1.3 \mathrm{M}$ (molar), 1.5M and 1.7M [36]. With the water content increased from $1.3 \mathrm{M}, 1.5 \mathrm{M}$ to $1.7 \mathrm{M}$, the yielding stresses were $\sigma=0.17 \mathrm{MPa}, 0.13$ $\mathrm{MPa}$ and $0.16 \mathrm{MPa}$, respectively. Practically, we can use the control of water content to design the stress and strain of DN hydrogels. Based on these analytical and experimental results, the yielding stress is determined by the water content. Both stress 
and strain are controlled by the water content in DN hydrogels, of which the mechanical properties are therefore designed by the water-induced swelling effect.

Table 4. Values of parameters used in equation (11) for DN hydrogels incorporated of various water contents.

\begin{tabular}{|c|c|c|c|c|c|c|c|c|c|c|}
\hline & $\lambda_{0}$ & $k_{r}$ & $B$ & $C$ & $v$ & $R T n_{1}(\mathrm{MPa})$ & $\frac{3 \xi}{N_{A} n_{1}}$ & $q_{a}$ & $E(\mathrm{MPa})$ & $L(\mathrm{MPa})$ \\
\hline $1.7 \mathrm{M}$ & 34 & 25 & 9.2 & 0.8 & 0.5 & 0.017 & 11.6 & 23 & 0.35 & -0.3 \\
\hline $1.5 \mathrm{M}$ & 32 & 28 & 9.6 & 0.9 & 0.55 & 0.006 & 9.7 & 25 & 0.27 & 0.005 \\
\hline $1.3 \mathrm{M}$ & 34 & 25 & 11.2 & 1 & 0.58 & 0.006 & 9.7 & 17 & 0.23 & -0.07 \\
\hline
\end{tabular}

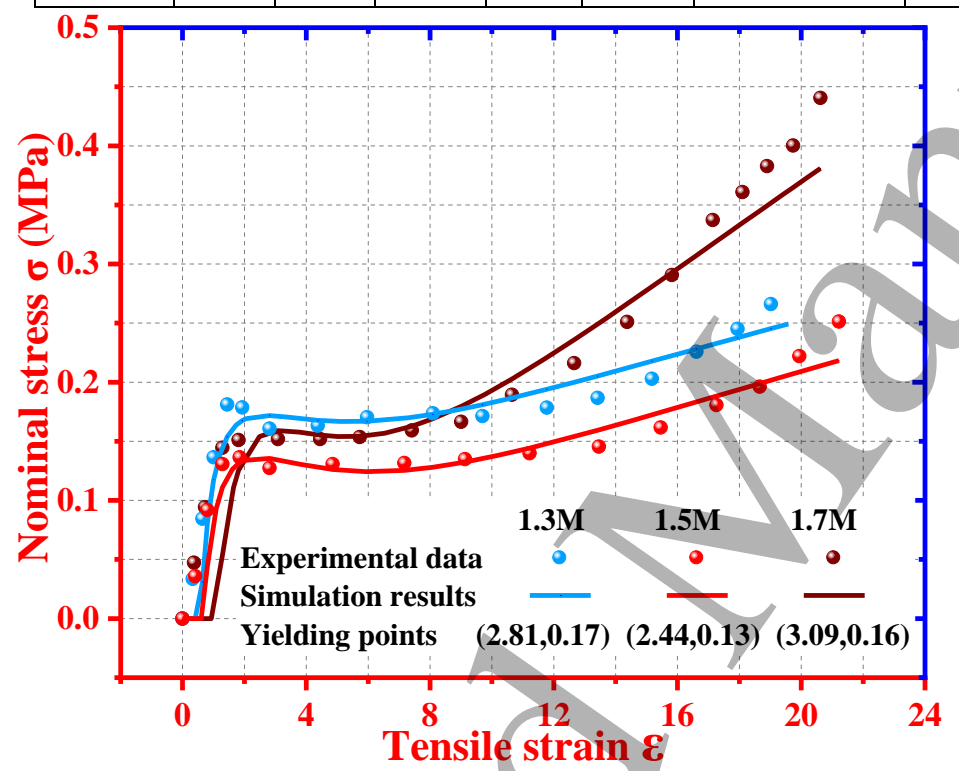

Figure 5. The comparison of simulation results and experimental data [36] for the stress as a function of tensile strain of DN hydrogel with various water contents.

\section{Stress-induced globule-to-coil phase transition in DN elastomers}

\subsection{Stress-strain constitutive relationship}

As discussed above, the working mechanism of water-induced globule-to-coil phase transition in DN hydrogels has been presented. Furthermore, scaling dynamics of stress-induced globule-to-coil phase transition in DN elastomers is necessary to investigate. The water-induced globule-to-coil phase transition is originated from the 
difference in Flory-Huggins interaction parameters $(\chi)$ of two networks with water in DN hydrogels. On the other hand, the stress-induced one is due to the difference in the mechanical properties of two networks. According to Flory-Huggins theory [38], the $\chi$ is a dynamic parameter and determined by the weight fraction of polymer $(\phi)$, degree of polymerization $(f(\xi))$ and temperature $(T)$,

$$
\begin{gathered}
\chi=f(\xi)\left(\frac{A}{T}+B \phi+C\right) \\
\Delta F=-\Delta F_{13}-\Delta F_{23}+\Delta F_{e l}^{m i c}+\Delta F_{m c}
\end{gathered}
$$

where subscripts 1, 2 and 3 denote two networks and stress within the DN elastomer, respectively, $\Delta F_{m c}$ is the mechanochemical free energy function, subscripts 13 and 23 denote the interactions between the two networks and stress, respectively.

According to equations (1), (2), (9) and (12), the constitutive stress-strain relationship of DN elastomers under uniaxial tension can therefore be obtained:

$$
\begin{aligned}
& \sigma=-\frac{\partial \Delta F}{\partial \lambda}=\frac{R \operatorname{Tn}_{1}}{2}\left[\frac{3\left(2-v_{13}-v_{23}\right) \lambda_{0}^{3}}{\lambda\left(\lambda^{3}-\lambda_{0}^{3}\right)}+3\left(v_{13}+v_{23}\right) \frac{3 \lambda^{2} \lambda_{0}^{3}}{\left(\lambda^{3}-\lambda_{0}^{3}\right)^{2}} \ln \frac{\lambda}{\lambda_{0}}\right. \\
& \left.-\left(f_{1}(\xi)+f_{2}(\xi)\right)\left(\frac{3 A}{T}+6 B \frac{\lambda_{0}^{3}}{\lambda^{3}}+3 C\right) \frac{\lambda_{0}^{3}}{\lambda^{4}}\right]+R T n_{1} \frac{3 \xi}{N_{A} n_{1}} \frac{\lambda}{\lambda_{0}^{2}}\left(1+\Delta V_{m i c}\right)^{2 / 3}+L
\end{aligned}
$$

To verify the applicability of proposed equation (13), the numerical results of the model are plotted and comparred with the experimental data [41] of $\mathrm{EA}_{0.5} \mathrm{MA}, \mathrm{EA}_{1} \mathrm{MA}$, $\mathrm{EA}_{2} \mathrm{MA}, \mathrm{EA}_{1} \mathrm{MA}_{2}, \mathrm{EA}_{0.5} \mathrm{MA}_{2}, \mathrm{EA}_{0.5} \mathrm{EA}$ and $\mathrm{EA}_{0.5} \mathrm{EA}_{2}$ (EA: ethyl acrylate and MA: methyl acrylate) DN elastomers with various component contents of two networks. There are three mole concentrations of cross-linker in the first network, i.e. $\mathrm{EA}_{0.5}$ is crosslinked at 1.45 mole percent (mol \%) of monomer, EA $\mathrm{A}_{1}$ two times more $(2.81$ mol \%), and $\mathrm{EA}_{2}$ four times more (5.81 mol \%). The single-network (SN), DN and 
triple-network $(\mathrm{TN})$ are used for $\mathrm{EA}_{1}, \mathrm{EA}_{1} \mathrm{MA}$ and $\mathrm{EA}_{1} \mathrm{MA}_{2}$. In the synthesis process, the first network is made from EA and cross-linker of butanediol diacrylate, and the second and third networks have been carried out with EA or MA. The parameters used in equation (13) are collected in Tables 5 and 6. As shown in Figure 6(a), the simulation curves fit well with the experimental data. And the mechanical stresses were $\sigma=4.98$ $\mathrm{MPa}$ and $\sigma=16.6 \mathrm{MPa}$ for the $\mathrm{EA}_{0.5} \mathrm{MA}$ and $\mathrm{EA}_{0.5} \mathrm{MA}_{2} \mathrm{DN}$ elastomers, respectively, at the temperature of $T=60^{\circ} \mathrm{C}$ and a given elongation ratio of $\lambda=2.2$. On the other hand, the stresses were $\sigma=1.97 \mathrm{MPa}$ and $\sigma=15.1 \mathrm{MPa}$ for the $\mathrm{EA}_{0.5} \mathrm{EA}$ and $\mathrm{EA}_{0.5} \mathrm{EA}_{2} \mathrm{DN}$ elastomers, respectively, at the temperature of $T=20^{\circ} \mathrm{C}$ and a given elongation ratio of $\lambda=2.2$. These analytical and experimental results reveal that the mechanical stress is significantly enhanced by the component content of second network (MA component or EA component) from $2.81 \mathrm{~mol} \%$ to $5.81 \mathrm{~mol} \%$ in the DN elastomer. The presence of second network is able to decrease the elastic component of the free energy per unit volume, resulting in the first network stretched further. On the other hand, the polymerization of second network is helpful to loosely connect the networks with each other and enables an essential stress transfer between the networks [41]. Therefore, the same analytical results have been verified by the experimental ones of $\mathrm{EA}_{1} \mathrm{MA}$ and $\mathrm{EA}_{1} \mathrm{MA}_{2} \mathrm{DN}$ elastomers, where the mechanical stress increases by a factor of up to 4.75 (from 5.92 MPa to 28.1 MPa) with an increase in the component content of second MA network from $2.81 \mathrm{~mol} \%$ to $5.81 \mathrm{~mol} \%$, as shown in Figure 6(b).

Furthermore, the effect of the first network on the mechanical behaviour of DN elastomer was investigated to identify the working mechanism behind experimental 
results. The analytical and experimental results of $\mathrm{EA}_{0.5} \mathrm{MA}, \mathrm{EA}_{1} \mathrm{MA}$ and $\mathrm{EA}_{2} \mathrm{MA} \mathrm{DN}$ elastomers have been plotted and compared in Figure 6(c). It was found that the maximum extensibility of DN elastomer was gradually decreased from $\lambda=2.44, \lambda$ $=1.93$ to $\lambda=1.63$, with an increase in the component content of first EA network from $1.45 \mathrm{~mol} \%, 2.81 \mathrm{~mol} \%$ to $5.81 \mathrm{~mol} \%$. These analytical results present that the first network is able to limit the maximum extensibility of DN elastomer, resulting in the maximum elongation ratio is therefore decreased with an increase in the component content of the first network. Finally, the proposed model was applied to characterize the mechanical behaviour of the DN elastomer under step-cycle loading-unloading, as shown in Figure 6(d). By comparison, it is found that the analytical results fit well with the experimental ones, and the proposed model is able to characterize the cyclically mechanical behaviours of the DN elastomers.

Table 5. Values of parameters used in equation (13) for Figures 6(a), 6(b) and 6(c), where $k_{r}=1000$.

\begin{tabular}{|c|c|c|c|c|c|c|c|}
\hline & $\mathrm{EA}_{0.5} \mathrm{MA}$ & $\mathrm{EA}_{1} \mathrm{MA}$ & $\mathrm{EA}_{2} \mathrm{MA}$ & $\mathrm{EA}_{1} \mathrm{MA}_{2}$ & $\mathrm{EA}_{0.5} \mathrm{MA}_{2}$ & $\mathrm{EA}_{0.5} \mathrm{EA}$ & $\mathrm{EA}_{0.5} \mathrm{EA}_{2}$ \\
\hline$A(K)$ & 133.4 & 133.4 & 133.4 & 133.4 & 133.4 & 117.4 & 117.4 \\
\hline$B$ & 2 & 1.92 & 1.92 & 1.92 & 1.92 & 1.92 & 1.92 \\
\hline$\left(v_{13}+v_{23}\right) / 2$ & 0.4 & 0.4 & 0.3 & 0.35 & 0.35 & 0.35 & 0.46 \\
\hline$R T n_{1}(\mathrm{MPa})$ & 0.51 & 0.2 & 0.11 & 0.52 & 0.116 & 0.14 & 0.18 \\
\hline $3 \xi / N_{A} n_{1}$ & 3.8 & 12 & 14 & 10.8 & 10.2 & 11 & 11 \\
\hline$q_{a}$ & 236 & 180 & 174 & 161 & 161 & 168 & 154 \\
\hline$E(\mathrm{MPa})$ & 0.546 & 1.769 & 1.77 & 1.484 & 0.793 & 0.511 & 1.07 \\
\hline$L_{1}(\mathrm{MPa})$ & -2.1 & -2.3 & -0.8 & -3.8 & -0.4 & -1.9 & -1.8 \\
\hline$f_{1}(\xi)$ & 0.354 & 0.353 & 0.263 & 0.333 & 0.343 & 0.635 & 0.494 \\
\hline$f_{2}(\xi)$ & $/$ & $/$ & $/$ & 1.067 & 1.457 & $/$ & 1.206 \\
\hline
\end{tabular}


Table 6. Values of parameters used in equation (13) for Figure 6(d), where $A=133.4 \mathrm{~K}$, $k_{r}=1000$ and $f_{1}(\xi)=0.408$.

\begin{tabular}{|c|c|c|c|c|c|c|c|c|}
\hline & $B$ & $\left(v_{13}+v_{23}\right) / 2$ & $R T n_{1}(\mathrm{MPa})$ & $3 \xi / N_{A} n_{1}$ & $q_{a}$ & $E(\mathrm{MPa})$ & $L_{1}(\mathrm{MPa})$ & $f_{2}(\xi)$ \\
\hline 1st cycle & 1.92 & 0.46 & 0.22 & 11 & 154 & 1.314 & -1.8 & 1.292 \\
\hline 2nd cycle & 2 & 0.47 & 0.26 & 10.3 & 178 & 0.87 & 2 & 1.393 \\
\hline 3rd cycle & 2 & 0.5 & 0.35 & 10 & 189 & 0.71 & 1.5 & 1.492 \\
\hline 4th cycle & 2 & 0.54 & 0.35 & 9.8 & 201 & 0.56 & 2 & 1.591 \\
\hline
\end{tabular}
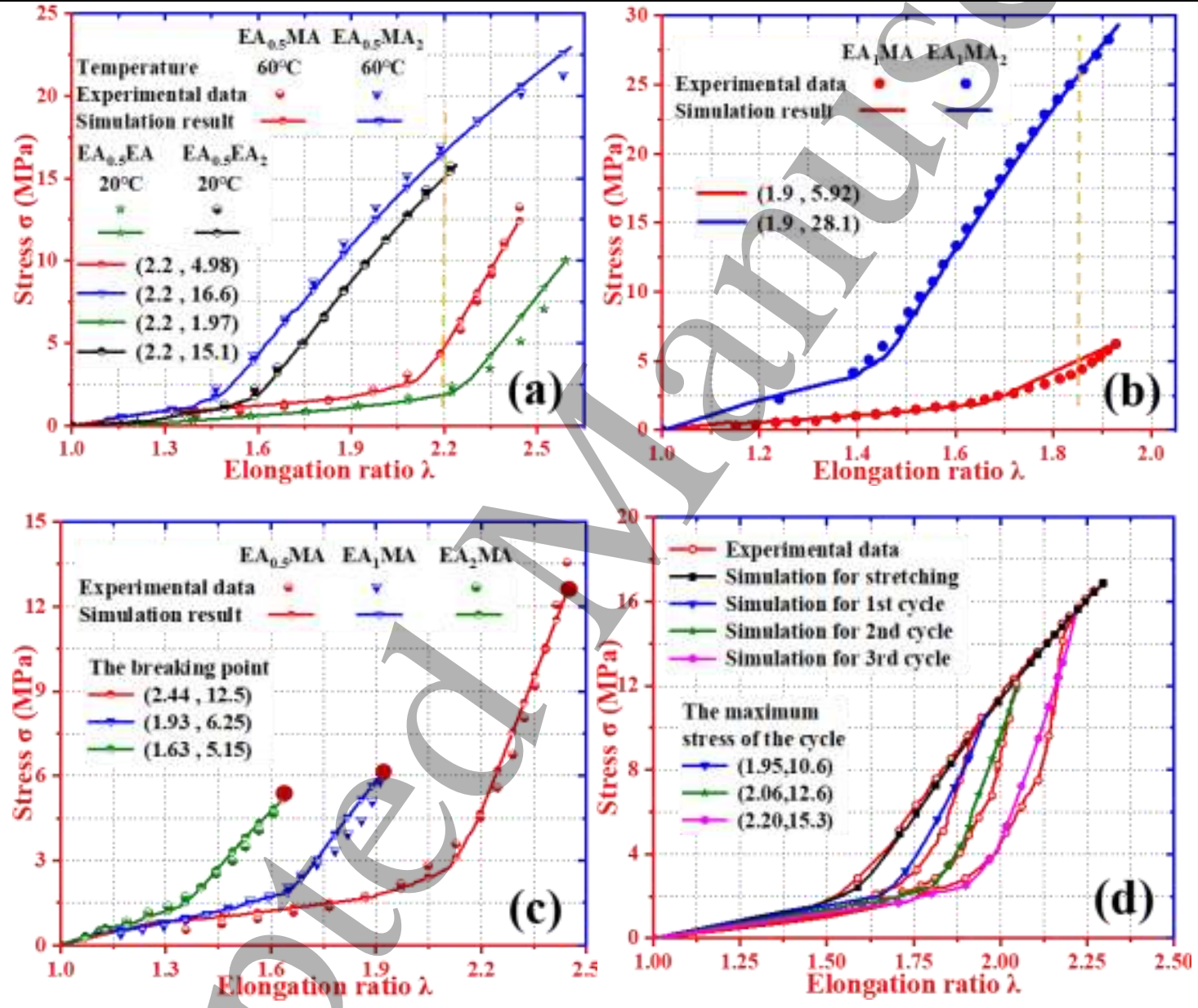

Figure 6. Comparison of analytical and experimental results [41] of the mechanical behaviours of DN/elastomers. (a) Mechanical stresses of DN elastomers as a function of elongation ratio at temperatures of $20^{\circ} \mathrm{C}$ and $60^{\circ} \mathrm{C}$. (b) Effect of component content of second network on the mechanical behaviour of DN elastomers. (c) Effect of component content of first network on the maximum extensibility of DN elastomers.

(d) Step-cycle loading-unloading curves of $\mathrm{EA}_{0.5} \mathrm{MA}_{2}$ elastomer at $60^{\circ} \mathrm{C}$. 


\subsection{Thermomechanical behaviour}

It is assumed that the weight fraction of first and second networks are equal to each other in the DN elastomer, i.e. $\phi_{1}=\phi_{2}=0.5$ and $\phi_{1}+\phi_{2}=1$, thus, the Maxwell equation of the DN elastomer can be re-written as [38],

$$
\sigma+\frac{\eta}{E_{2}} \frac{d \sigma}{d t}=E_{1} \varepsilon+\left(E_{1}+E_{2}\right) \frac{\eta}{E_{2}} \frac{d \varepsilon}{d t}
$$

where $\eta$ is the viscosity, $E_{1}$ and $E_{2}$ are the moduli of the first and second networks, respectively, and $t$ is time. $\frac{d \sigma}{d t}$ and $\frac{d \varepsilon}{d t}$ are the stress rate and strain rate, respectively.

Based on the Maxwell principle [38], equation (13) can be rewritten by introducing a parameter $\hat{\varepsilon} e^{i \omega t}$ to replace $\varepsilon$,

$$
\sigma+\frac{\eta}{E_{1}} \frac{d \sigma}{d t}=\hat{\varepsilon} e^{i \omega t}\left[E_{2}+\left(E_{1}+E_{2}\right) \frac{\eta}{E_{1}} i \omega\right]
$$

where $\tau=\frac{\eta}{E_{1}}, \hat{\varepsilon}$ is the referenced strain, $\omega$ is the loading frequency and $i=\sqrt{-1}$. Thus, the storage and the loss moduli of the DN elastomer can be obtained [38], e.g.,

$$
\left\{\begin{array}{l}
E^{\prime}=\frac{E_{2}+\tau^{2} \omega^{2}\left(E_{1}+E_{2}\right)}{1+\tau^{2} \omega^{2}} \\
E^{\prime \prime}=\frac{E_{1} \tau \omega}{1+\tau^{2} \omega^{2}}
\end{array}\right.
$$

Both the moduli of the first and second networks are different from their original ones due to the changes in their weight fractions, which are resulted from the sacrificial bonds of first network, i.e. $\bar{E}_{1}=E_{1} \phi_{1}$ and $\bar{E}_{2}=E_{2} \phi_{2}$. Here, the relaxation time of the newly formed DN elastomer can be expressed as,

$$
\bar{\tau}=\frac{\eta}{\bar{E}_{2}}=\frac{\eta}{E_{2} \phi_{2}}=\frac{1}{\phi_{2}} \cdot \frac{\eta}{E_{2}}=\frac{1}{\phi_{2}} \cdot \tau
$$


Substituting equation (15) into (14), we can obtain,

$$
\left\{\begin{array}{l}
E^{\prime}=\frac{\phi_{1}^{2} \phi_{2} \bar{E}_{2}+\bar{\tau}^{2} \omega^{2}\left(\bar{E}_{1} \phi_{1}+\bar{E}_{2} \phi_{2}\right)}{\phi_{1}^{2}+\bar{\tau}^{2} \omega^{2}} \\
E^{\prime \prime}=\frac{\bar{E}_{1} \bar{\tau} \omega}{1+\frac{1}{\phi_{1}^{2}} \bar{\tau}^{2} \omega^{2}}
\end{array}\right.
$$

According to the time-temperature equivalence principle [42], the constitutive relationship of time $(1 / \omega)$ and transition temperature $\left(T_{g}\right)$ can be obtained,

$$
\left\{\begin{array}{l}
E^{\prime}\left(T_{g}, \omega\right)=E^{\prime}\left(T_{g}^{\prime}, a_{T} \omega\right) \\
E^{\prime \prime}\left(T_{g}, \omega\right)=E^{\prime \prime}\left(T_{g}^{\prime}, a_{T} \omega\right)
\end{array}\right.
$$

where $T_{g}^{\prime}$ is the referenced transition temperature, $a_{T}$ is a shift factor $\left(a_{T} \omega=\omega^{f_{T}}\right.$ in logarithmic sense), which can be determined using the temperature based on the Williams-Landel-Ferry (WLF) equation [42],

$$
\lg a_{T}=\frac{-17.44\left(T_{g}^{\prime}-T_{g}\right)}{51.6+\left(T_{g}^{\prime}-T_{g}^{\prime}\right)}
$$

According to the equations (18), (19) and (20), the glass transition temperature $\left(T_{g}\right)$ can be expressed as,

$$
T_{g}=T_{g}^{\prime}+\frac{C_{2} 10^{\phi_{1}^{2} / \bar{\tau}^{2}}}{C_{1}+10^{\phi_{1}^{2} / \bar{\tau}^{2}}}
$$

The analytical results of storage and loss moduli of the DN elastomer have been plotted as a function of frequency, and the results are shown in Figure 7. The parameters used in the equation (19) are $\bar{E}_{1}=2 \mathrm{MPa}, \bar{E}_{2}=0.2 \mathrm{MPa}, \bar{\tau}=1.0 \times 10^{-6} \mathrm{~S}$ and $a_{T}=1$. Figures 7(a), 7(b), 7(c) and 7(d) show the relationships among storage moduli, loss moduli and loading frequency for the DN elastomer with various weight fractions of the first network of $\phi_{1}=0.2, \phi_{1}=0.4, \phi_{1}=0.6$ and $\phi_{1}=0.8$, respectively. Furthermore, the analytical results of storage moduli as a function of weight fraction of the first 
network and those of loss moduli are also plotted and compared as shown in Figures 7(e) and 7(f), respectively. Both the storage and loss moduli of the DN elastomer are increased for the increased weight fraction of the first network.
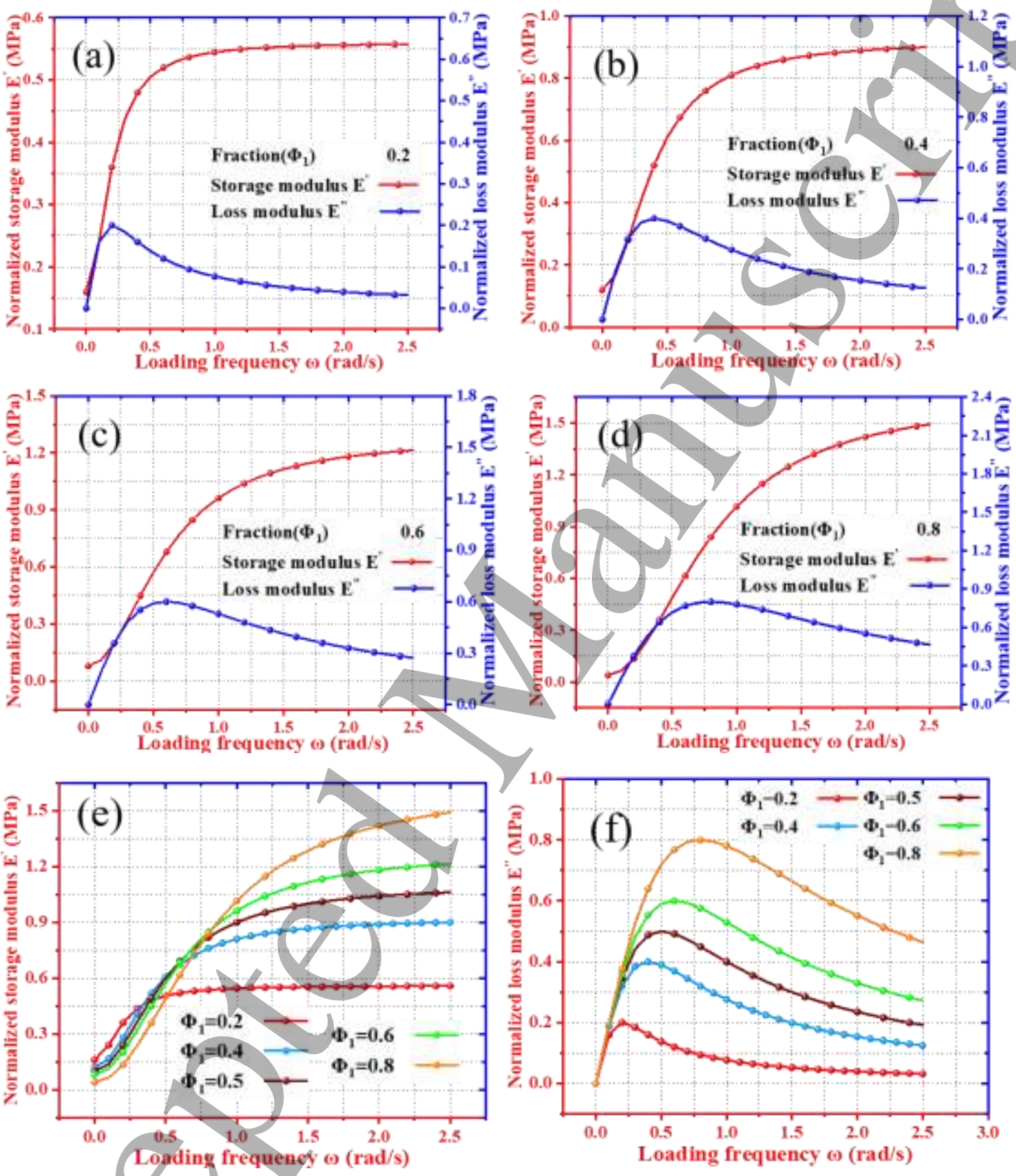

Figure 7. Analytical results of storage and loss moduli as a function of loading frequency at different weight fractions of first network. (a) $\phi_{1}=0.2$. (b) $\phi_{1}=0.4$. (c) $\phi_{1}$ $=0.6$ and (d) $\phi_{1}=0.8$. (e) Analytical results of storage moduli and (f) tangent delta as a function of loading frequency at $\phi_{1}=0.2,0.4,0.5,0.6$ and 0.8 , respectively. 
To verify the proposed models in equations of (19), (20), (21) and (22), the analytical results were compared with the experimental data [43], which are shown in Figure 8. Experimental data of poly(phenyl ether acrylate-co-isobornyl acrylate) (P(PEA-coIBA)) and poly(methyl ether acrylate-co-isobornyl acrylate) (P(MEA-co-IBA)) DN elastomers [43] were used to compare with the analytical results of storage and loss moduli as shown in Figure 8(a), and the parameters used in equations (19), (20) and (21) are listed in Table 7. The analytical results of glass transition temperature as a function of weight fraction of first network were plotted in Figure 8(b), and the parameters used in the equation (22) were listed in Table 8. It is found that the simulation results obtained from our model fit well with the experimental data, and the thermomechanical behaviours of DN elastomers have been well described using this new model. Furthermore, these simulation results yerified that the proposed models in equations of (19), (20), (21) and (22) provided effective approaches to design the thermomechanical behaviours of DN elastomers to achieve their designable mechanical performances.

Table 7. Values of parameters used in equations (19), (20) and (21).

\begin{tabular}{|c|c|c|c|c|c|}
\hline & $\bar{E}_{1}(\mathrm{MPa})$ & $\bar{\tau}\left(10^{-6} \mathrm{~s}\right)$ & $\phi_{1}$ & $f_{T}$ & $\bar{E}_{2}(\mathrm{MPa})$ \\
\hline The storage modulus & 149.99 & 0.1265 & 0.4 & 0.35 & 0.083 \\
\hline The loss modulus & 77.47 & 0.1265 & 0.4 & 0.53 & $/$ \\
\hline
\end{tabular}

Table 8. Values of parameters used in equation (22).

\begin{tabular}{|l|c|c|c|c|}
\hline & $C_{1}$ & $C_{2}$ & $\bar{\tau}\left(10^{-6} \mathrm{~s}\right)$ & $T_{g}^{\prime}(\mathrm{K})$ \\
\hline P(PEA-co-IBA) & 16.1 & 76.4 & 0.5 & 279 \\
\hline P(MEA-co-IBA) & 18.2 & 118.2 & 0.5 & 237.5 \\
\hline
\end{tabular}



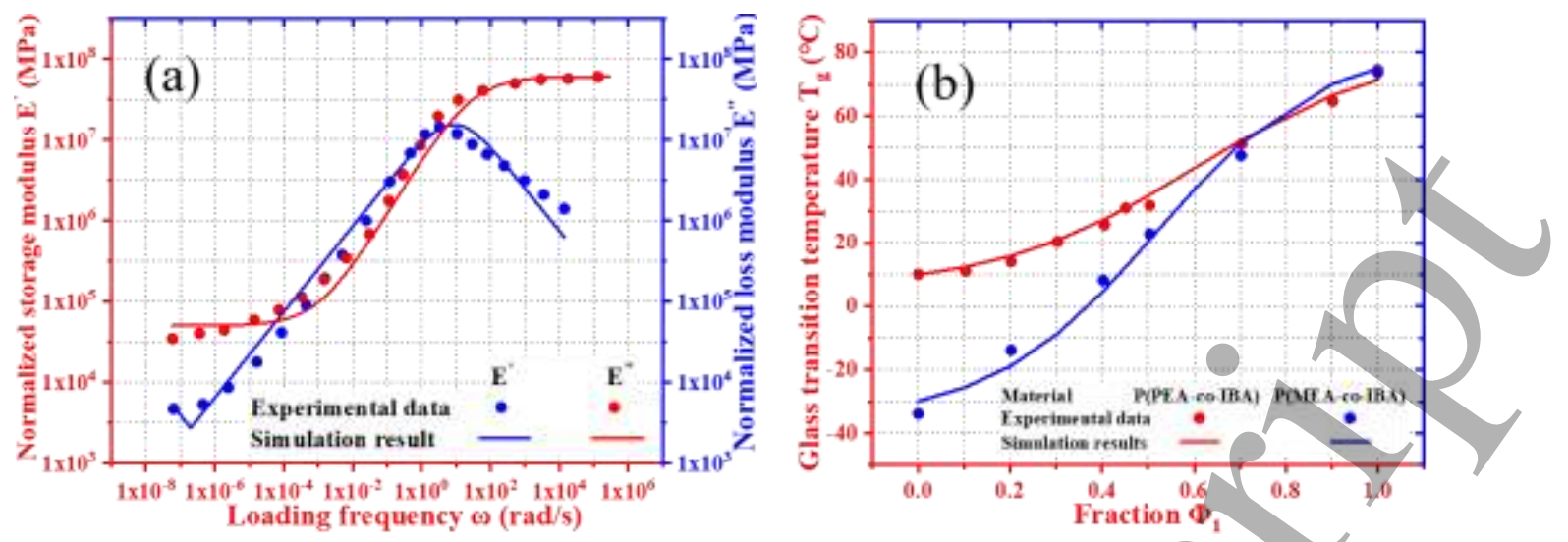

Figure 8. (a) Comparisons of storage and loss moduli as a function of loading frequency between the experimental data [43] and simulation results using equation (19), (20) and (21). (b) Comparison of glass transition temperature as a function of volume fraction $\left(\phi_{1}\right)$ of DN elastomers between the experimental data [43] and simulation results using equation (22).

\section{Conclusion}

In this study, we propose a scaling model to study the mechanical and thermomechanical behaviours of DN hydrogels, in which network undergoes swellinginduced and stress-induced globule-to-coil transition. Analytical results provide an effective approach to estimate and quantitatively separate the dependence of the stressstrain relationship on the free energy, globule radius, water content and component fraction. Globule radius and stress-induced globule-to-coil transition are critical parameters to restrict the extension of network in hydrogels, resulting in the loading efficiency and stretchable strength efficiently improved. Finally, the proposed models have been verified using the experimental results, and good agreements between the simulation results and the experimental ones have been achieved. We expect this study provides an effective guidance on designing advanced DN hydrogels and elastomers with ultra-high stretchable and strength capabilities, which can be utilized in practical applications of soft robotics and artificial muscle. 


\section{Acknowledgements}

This work was financially supported by the National Natural Science Foundation of China (NSFC) under Grant No. 11672342 and 11725208. 


\section{References}

[1] Johnson J A, Turro N J, Koberstein J T and Mark J E 2010 Some hydrogels having novel molecular structures Prog. Polym. Sci. 35 332-7

[2] Salahuddin B, Warren H and Spinks G M 2020 Thermally actuated hydrogel bead based braided artificial muscle Smart Mater. Struct. 29055042

[3] Hamedi H, Moradi S, Hudson S M and Tonelli A E 2018 Chitosan based hydrogels and their applications for drug delivery in wound dressings: A review Carbohyd. Polym. 199 445-60

[4] Kang Y Woo, Woo J, Lee H R and Sun J Y 2019 A mechanically enhanced electroactive hydrogel for $3 \mathrm{D}$ printing using a multileg long chain crosslinker Smart Mater. Struct. 28095016

[5] Lin P, Ma S H, Wang X L and Zhou F 2015 Molecularly engineered dualcrosslinked hydrogel with ultrahigh mechanical strength, toughness, and good self-recovery Adv. Mater. 27 2054-9

[6] Hu Y, Du Z S, Deng X L, Wang T, Yang Z H, Zhou W Y and Wang C Y 2016 Dual physically cross-linked hydrogels with high stretchability, toughness, and good self-recoverability Macromolecules 49 5660-8

[7] Dai X Y, Zhang Y Y, Gao L N, Bai T, Wang W, Cui Y L and Liu W G 2015 A mechanically strong, highly/ stable, thermoplastic, and self-healable supramolecular polymer hydrogel Adv. Mater. 27 3566-71

[8] Yuk H W, Zhang T, Lin S T, Parada G A and Zhao X H 2016 Tough bonding of hydrogels to diverse non-porous surfaces Nat. Mater. 15 190-6

[9] Gong J P 2010 Why are double network hydrogels so tough? Soft Matter 6 258390

[10] Yue Y F, Li X F, Kurokawa T, Haque Md A and Gong J P 2016 Decoupling dualstimuli responses in patterned lamellar hydrogels as photonic sensors J. Mater. Chem. B. 4 4104-9 
[11] Xing Z M, Wang C L, Yan J, Zhang L, Li L and Zha L S 2011 Dual stimuli responsive hollow nanogels with IPN structure for temperature controlling drug loading and $\mathrm{pH}$ triggering drug release Soft Matter 7 7992-7

[12] Tokarev I and Minko S 2009 Stimuli-responsive hydrogel thin films Soft Matter 5 $511-24$

[13] Yang C, Liu Z, Chen C, Shi K, Zhang L, Ju X J, Wang W, Xie R and Chu L Y, 2017 Reduced graphene oxide-containing smart hydrogels with excellent electroresponse and mechanical properties for soft actuators ACS Appl. Mater. Inter. 9 $15758-67$

[14] Fuhrer R, Athanassiou E K, Luechinger N A and Stark W J 2009 Crosslinking metal nanoparticles into the polymer backbone of hydrogels enables preparation of soft, magnetic field-driven actuators with muscle-like flexibility Small 5 383-8

[15] Zheng W J, An N, Yang J H, Zhou J X and Chen Y M 2015 Tough Alalginate/Poly(N-isopropylacrylamide) hydrogel with tunable LCST for soft robotics ACS Appl. Mater. Inter. 7 1758-64

[16] Webber R E, Creton C, Brown H R and Gong J P 2007 Large strain hysteresis and mullins effect of tough double-network hydrogels Macromolecules 40 2919-27

[17] Jang S S, Goddard W A and Kalani M Y S 2007 Mechanical and transport properties of the poly (ethylene oxide)-poly (acrylic acid) double network hydrogel from molecular dynamic simulations J. Phys. Chem. B. 111 1729-37

[18] Kwon H J, Yasuda K, Ohmiya Y, Honma K, Chen Y M and Gong J P 2010 In vitro differentiation of chondrogenic ATDC5 cells is enhanced by culturing on synthetic hydrogels with various charge densities Acta Biomater. 6 494-501

[19] Gong J P, Katsuyama Y, Kurokawa T and Osada Y 2013 Double-network hydrogels with extremely high mechanical strength Adv. Mater. 15 1155-8

[20]Zhai D and Zhang H 2013 Investigation on the application of the TDGL equation in macromolecular microsphere composite hydrogel Soft Matter 9 820-5 
[21]Lu H B, Shi X J, Yu K and Fu Y Q 2019 A strategy for modelling mechanochemically induced unzipping and scission of chemical bonds in doublenetwork polymer composite Compo. Part B-Eng. 165 456-66

[22]Koetting M C, Peters J T, Steichen S D and Peppas N A 2015 Stimulus-responsive hydrogels: Theory, modern advances, and applications Mat. Sci. Eng. R 93 1-49

[23]Hong W, Zhao X H and Suo Z G 2010 Large deformation and electrochemistry of polyelectrolyte gels J. Mech. Phys. Solids 58 558-77

[24] Marcombe R, Cai S Q, Hong W, Zhao X H, Lapusta Y and Suo Z G 2010 A theory of constrained swelling of a pH-sensitive hydrogel Soft Matter 6 784-93

[25]Li J Y, Suo Z G and Vlassak J J 2014 A model of ideal elastomeric gels for polyelectrolyte gels Soft Matter $102582-90$

[26]Basak D and Ghosh S 2013 pH-regulated controlled swelling and sustained release from the core functionalized amphiphilic block copolymer micelle ACS Macro Lett. $2799-804$

[27] Zhang H J, Sun T L, Zhang A K, Ikura Y, Nakajima T, Nonoyama T, Kurokawa T, Ito O, Ishitobi H and Gong J P 2016 Tough physical double-network hydrogels based on amphiphilic triblock copolymers Adv. Mater. 28 4884-90

[28] Na Y H, Tanaka Y, Kawauchi Y, Furukawa H, Sumiyoshi T and Gong J P 2006 Necking phenomenon of double-network gels Macromolecules 39 4641-5

[29] Nakajima T, Kurokawa T, Ahmed S, Wu W L and Gong J P 2013 Characterization of internal fracture process of double network hydrogels under uniaxial elongation Soft Matter 9 1955-66

[30] Zheng W J, Liu Z Q, Xu F, Gao J, Chen Y M, Gong J P and Osada Y 2015 In vitro platelet adhesion of PNaAMPS/PAAm and PNaAMPS/PDMAAm doublenetwork hydrogels Macromol. Chem. Phys. 216 641-9 
[31] Matsuda T, Nakajima T, Fukuda Y, Hong W, Sakai T, Kurokawa T, Chung U I and Gong J P 2016 Yielding criteria of double network hydrogels Macromolecules 49 $1865-72$

[32] Dopieralski P, Ribas-Arino J, Anjukandi P, Krupicka M and Marx D 2017 Unexpected mechanochemical complexity in the mechanistic scenarios of disulfide bond reduction in alkaline solution Nat. Chem. 9 164-70

[33]Zhong D M, Xiang Y H, Yin T H, Yu H H, Qu S X and Yang W 2019A physicallybased damage model for soft elastomeric materials with anisotropic Mullins effect Int. J. Solids Struct. 176-177 121-34

[34] Diesendruck C E, Peterson G I, Kulik H J, Kaitz J A, Mar B D, May P A, White S R, Martínez T J, Boydston A J and Moore J S 2014 Mechanically triggered heterolytic unzipping of a low-ceiling-temperature polymer Nat. Chem. 6 623-8

[35] Nakajima T, Takedomi N , Kurokawa T, Furukawa H and Gong J P 2010 A facile method for synthesizing free-shaped and tough double network hydrogels using physically crosslinked poly(vinyl alcohol) as an internal mold Polym. Chem. $1693-$ 7

[36]Ahmed S, Nakajima T, Kurokawa T, Haque M A and Gong J P 2014 Brittle-ductile transition of double network hydrogels: Mechanical balance of two networks as the key factor Polymer 55 914-23

[37]Ilyas M, Haque M A, Yue Y, Kurokawa T, Nakajima T, Nonoyama T and Gong J P 2017 Water-triggered ductile-brittle transition of anisotropic lamellar hydrogels and effect of confinement on polymer dynamics Macromolecules 50 8169-77

[38]He M J, Zhang H D, Chen W X and Dong X X 2006 Polymer Physics (Shanghai: Fudan Press) 
[39]Gennes P G 1979 Scaling Concepts in Polymer Physics (Ithaca and London: Cornell University Press)

[40]Xu Z L 1991 Elasticity Mechanics (Beijing: Higher Education Press)

[41]Ducrot E, Chen Y L, Bulters M, Sijbesma R P and Creton C 2014 Toughening elastomers with sacrificial bonds and watching them break Science 344 187-9

[42] Williams M L, Landell R F and Ferry J D 1955 The temperature dependence of relaxation mechanisms in amorphous polymers and other glass-forming liquids $J$. Am. Chem. Soc. 77 3701-7

[43]Chen L, Sun T L, Cui K P, King D R, Kurokawa T, Saruwatari Y and Gong J P 2019 Facile synthesis of novel elastomers with tunable dynamics for toughness, self-healing and adhesion J. Mater. Chem. A 7 17334-44 


\section{Tables caption}

Table 1. Values of parameters used in equation (11) for Figure 2.

Table 2. Values of parameters used in equation (11) for Figure 3.

Table 3. Values of parameters used in equation (11) for DN0.1, PVA-DN0.1, DN0.6 and PVA-DN0.6, where $C=0.5, \quad r_{a}=2$ and $q_{b}=10$.

Table 4. Values of parameters used in equation (11) for DN hydrogels incorporated of various water contents.

Table 5. Values of parameters used in equation (13) for Figures 6(a), 6(b) and 6(c), where $k_{r}=1000$.

Table 6. Values of parameters used in equation (13) for Figure 6(d), where $A=133.4 \mathrm{~K}$, $k_{r}=1000$ and $f_{1}(\xi)=0.408$.

Table 7. Values of parameters used in equations (19), (20) and (21).

Table 8. Values of parameters used in equation (22). 


\section{Figures caption}

Figure 1. Schematic diagram of the globule-to-coil transition behaviours of the two networks in DN hydrogel.

Figure 2. Simulation results of stress as a function of elongation ratio of DN hydrogel, at given $r_{b} / r_{a}$ ratios of $5,6,7,8$ and 9 .

Figure 3. Analytical results of stress as a function of elongation ratio, at different molar fractions of water, i.e. $n_{1}=1 \times 10^{-4}, 1.5 \times 10^{-4}, 2 \times 10^{-4}, 2.5 \times 10^{-4}$ and $3 \times 10^{-4} \mathrm{~mol}$.

Figure 4. Comparisons between numerical results using equation (11) and experimental data [31] of the DN and PVA-DN hydrogels.

Figure 5. The comparison of simulation results and experimental data [36] for the stress as a function of tensile strain of DN hydrogel with various water contents.

Figure 6. Comparison of analytical and experimental results [41] of the mechanical behaviours of DN elastomers. (a) Mechanical stresses of DN elastomers as a function of elongation ratio at temperatures of $20^{\circ} \mathrm{C}$ and $60^{\circ} \mathrm{C}$. (b) Effect of component content of second network on the mechanical behaviour of DN elastomers. (c) Effect of component content of first network on the maximum extensibility of DN elastomers.

(d) Step-cycle loading-unloading curves of $\mathrm{EA}_{0.5} \mathrm{MA}_{2}$ elastomer at $60^{\circ} \mathrm{C}$.

Figure 7. Analytical results of storage and loss moduli as a function of loading frequency at different weight fractions of first network. (a) $\phi_{1}=0.2$. (b) $\phi_{1}=0.4$. (c) $\phi_{1}$ $=0.6$ and (d) $\phi_{1}=0.8$. (e) Analytical results of storage moduli and (f) tangent delta as a function of loading frequency at $\phi_{1}=0.2,0.4,0.5,0.6$ and 0.8 , respectively.

Figure 8. (a) Comparisons of storage and loss moduli as a function of loading frequency 
between the experimental data [43] and simulation results using equation (19), (20) and (21). (b) Comparison of glass transition temperature as a function of volume fraction $\left(\phi_{1}\right)$ of DN elastomers between the experimental data [43] and simulation results using equation (22). 